\title{
Diagnostic utility of alarm features in predicting malignancy in patients with dyspeptic symptoms
}

\author{
Anurag Shetty ${ }^{1}$ (D) $\cdot$ Girisha Balaraju ${ }^{2} \cdot$ Shiran Shetty ${ }^{2} \cdot$ Cannanore Ganesh Pai $^{2}$
}

Received: 28 August 2020 / Accepted: 28 January 2021 / Published online: 8 April 2021

(C) The Author(s) 2021

\begin{abstract}
Background Clinical features are of modest benefit in determining the etiology of dyspepsia. Dyspeptic patients with alarm features are suspected to have malignancy; but the proportions of patients and true cutoff values of various quantitative parameters in predicting malignancy are explored to a lesser extent.

Methods This is a prospective observational study of consecutive patients undergoing esophagogastroduodenoscopy (EGD) for dyspeptic symptoms. Patients' alarm features and clinical details were recorded in a predesigned questionnaire. The diagnostic accuracy of alarm features in predicting malignancy was studied.

Results Nine hundred patients, 678 (75.3\%) males, with a mean (standard deviation [SD]) age of 44.6 (13.54) years were enrolled. Commonest indication for EGD was epigastric pain in $614(68.2 \%)$ patients. Dyspepsia was functional in 311 (34.6\%) patients. EGD revealed benign lesions in 340 (37.8\%) and malignancy in 50 (5.5\%) patients. Among the malignant lesions, gastric malignancy was present in $28(56 \%)$ and esophageal malignancy in $20(40 \%)$ patients. Alarm features were present in $206(22.9 \%)$, out of which malignant lesions were seen in $46(22.3 \%)$ patients. Altogether, the alarm features had a sensitivity of $92 \%$ and specificity of $81.2 \%$ for predicting malignancy. The sensitivity and specificity for weight loss were $76 \%$ and $90.8 \%$, while that of abdominal mass were $10 \%$ and $99.9 \%$ respectively. Based on receiver operating characteristic curve, the optimal age for screening of malignancy was 46.5 years in this population.

Conclusions Patients of age group 40 to 49 years with dyspeptic alarm symptoms (predominant weight loss) need prompt endoscopy to screen for malignancy. The alarm features are inexpensive screening tools, found to be useful in India, and should be utilized in countries with similar healthcare conditions and disease epidemiology.
\end{abstract}

Keywords Abdominal pain - Alarm symptoms · Diagnostic accuracy $\cdot$ Dyspepsia $\cdot$ Endoscopy $\cdot$ Evaluation $\cdot$ India Management $\cdot$ Risk factors $\cdot$ Surveillance $\cdot$ Upper gastrointestinal malignancy

\section{Introduction}

Dyspepsia presents with persistent or recurrent pain or discomfort in the upper abdomen in addition to other gastrointestinal (GI) symptoms [1]. It is the commonest condition seen by gastroenterologists on a daily basis in which organic causes are seen only in a few [2]. Its prevalence varies from $25 \%$ to $40 \%$ in

Anurag Shetty

shettyanurag@gmail.com

1 Department of Gastroenterology and Hepatology, Kasturba Medical College, Mangalore, Manipal Academy of Higher Education, Manipal 575 003, India

2 Department of Gastroenterology and Hepatology, Kasturba Medical College, Manipal, Manipal Academy of Higher Education, Manipal 576 104, India western countries [3] while the prevalence in Asian population is $8 \%$ to $30 \%$ [4]. It leads to considerable impact on treatment as the estimated direct annual cost incurred in treating dyspepsia is over $\$ 12$ billion in the USA [5]. Multiple conditions may mimic dyspepsia or may underlie dyspepsia, including gastroesophageal reflux disease (GERD), peptic ulcer, and most importantly GI malignancy, which contribute to $1 \%$ to $3 \%$ of all patients with dyspepsia $[6,7]$. In more than $50 \%$ of the patients with uninvestigated dyspepsia, nor obvious organic cause is found (a condition called functional dyspepsia) [8]. Esophagogastroduodenoscopy (EGD) helps in the initial diagnosis but numerous attempts at recognizing patients most likely to benefit from endoscopic evaluation have not been successful. The salient feature of any empirical treatment for dyspepsia is to minimize the risk of missing a GI malignancy at a treatable stage. Alarm features and age 


\section{Bullet points of the study highlights}

\section{What is already known?}

- Dyspepsia is widely prevalent in India.

- The literature for the proportion of patients and true cutoff values in predicting malignancy with alarm features remains little explored in real-world setting.

\section{What is new in this study?}

- Among the alarm features, weight loss is one of the most predominant factors with good sensitivity and specificity.

- The optimal age to begin screening for malignancy in dyspepsia patients in India seems to be 46.5 years.

\section{What are the future clinical and research implications of the study findings?}

- There is a need to identify appropriate alarm features in dyspeptic patients and their presence need prompt assessment.

- Further research to assess the cost effectiveness analysis of routine endoscopy vs. the use of only alarm features as a screening tool in dyspeptic patients requiring endoscopy is needed.

were of limited value for predicting malignancy in patients with dyspepsia from Asian countries [9]. Dyspeptic patients with alarm features are suspected to have malignancy; but the proportion of patients and true cutoff values of various quantitative parameters in predicting malignancy are explored to a lesser extent in Asian countries. The diagnostic utility of dyspeptic alarm symptoms in predicting who has malignancy is unclear [9]. So, this study is aimed to determine the spectrum of malignancies in patients with dyspepsia and frequency of alarm features and to evaluate the diagnostic accuracy of these alarm features in predicting upper GI malignancy.

\section{Methods}

This is a prospective observational study of consecutive patients undergoing EGD for dyspeptic symptoms at a tertiary care teaching hospital from November 2013 to December 2014. The study protocol was approved by the Institutional Ethics Committee (Approval Number IEC 547/2013). A written informed consent was obtained from all the patients prior to their enrolment. This study was conducted in accordance with Good Clinical Practice and in a manner to conform to the Helsinki Declaration of 1975, as revised in 2000 and 2008 concerning human rights. The inclusion criteria was dyspepsia of greater than 4 weeks (ROME III criteria) in adult patients (>18 year) [10]. The exclusion criteria were pregnancy, cholelithiasis, pancreatitis, history of surgery of the esophagus or stomach, previously diagnosed cirrhosis of the liver, treated malignancy, use of proton pump inhibitors daily for $>4$ weeks duration, predominant heartburn, anemia weight loss, predominant dysphagia, and refusal of consent. Relevant clinical details regarding concomitant medication, addictions, height, weight, body mass index, and alarm features like anemia, significant weight loss defined as unintended weight loss of more than $10 \%$ over 3 months, abdominal mass palpable, supraclavicular lymph nodes, persistent vomiting, GI bleeding, dysphagia, and family history of malignancies were recorded in a predesigned questionnaire.

Standard EGD (GIF-N180 [Olympus, Tokyo, Japan]) was performed by experienced gastroenterologists in all the patients under local anesthesia and endoscopic findings were recorded. In case of suspected malignancy, multiple biopsy specimens were taken from the suspected lesion and sent for histological confirmation. The biopsy specimen was interpreted by an expert pathologist. Treatment for dyspepsia was done as per appropriate guidelines [11].

\section{Statistical analysis}

The Statistical Package for Social Sciences version 16 (SPSS Inc., Chicago, IL, USA) for windows was used for the statistical analysis. Descriptive statistics were used as appropriate. The Chi-square test was used for categorical data, while analysis of variance (ANOVA) and Student's $t$ test were used for continuous data. The diagnostic values of individual alarm feature, 
Table 1 Baseline characteristics of patients

Number $(\%)$

\section{Parameter}

Total number of patients

Age, mean (SD) in year

Symptom duration in months, median (range)

$900(100)$

44.6 (13.54)

$8(1-360)$

Gender, M:F

BMI $\left(\mathrm{kg} / \mathrm{m}^{2}\right)$, mean (SD)

678 (75.3), 3.05:1

Smoking

$24.5(4.2)$

$152(16.9)$

Alcoholism

$219(24.3)$

Tobacco use

$106(11.8)$

Comorbid conditions

Hypertension

$89(9.9)$

Diabetes mellitus

$54(6)$

Ischemic heart disease

$22(2.4)$

Bronchial asthma/COPD

$14(1.5)$

$7(0.8)$

Presenting alarm features ${ }^{\#}$

Weight loss

$116(12.9)$

$52(5.8)$

40 (4.4)

44 (4.9)

$6(0.7)$

$13(1.4)$

$79(8.9)$

$4(0.4)$

Supraclavicular lymph nodes

Indication for EGD in addition to alarm features ${ }^{\#}$

Epigastric pain

$614(68.2)$

Epigastric burning

$569(63.2)$

"Some patients may have more than one presenting /alarm symptoms $S D$ standard deviation, $M$ male, $F$ female, $B M I$ body mass index, $C O P D$ chronic obstructive pulmonary disease, $E G D$ esophagogastroduodenoscopy

including sensitivity, specificity, positive predictive value (PPV), and negative predictive value (NPV), were calculated. Based on this, area under the receiver operating characteristic (ROC) curve was used to find out an optimal age for screening of malignancy. A $p$-value of $<0.05$ was considered to be statistically significant.

\section{Results}

\section{Study population}

Nine hundred patients, $678(75.3 \%)$ males, with a mean (SD) age of 44.6 (13.54) years with majority (648 [72\%]) being in the age group of $25-55$ years were included. Hypertension was the most prevalent comorbidity seen in $89(9.9 \%)$ patients (Table 1$)$. The commonest indication for EGD was epigastric pain in 614 (68.2\%) patients. Dyspepsia was functional in 311 (34.6\%) patients. EGD revealed benign lesions in 340 (37.8\%) and malignancy in $50(5.5 \%)$ patients. Among the histologically confirmed malignant lesions, gastric malignancy was present in 28 (56\%), esophageal malignancy in 20 (40\%), and duodenal malignancy in $2(8 \%)$ patients. On analyzing the relationship of duration of dyspepsia with malignancy, it was found that the patients with malignancy had a mean (SD) duration of dyspepsia of 5.14 (16.84) months compared to $26.8(45.7 \%)$ in patients without malignancy which was statistically significant $(p<0.001)$. Patients with lower hemoglobin levels had higher proportion of malignancy. 13/61 (21.3\%) patients with hemoglobin levels $<10 \mathrm{~g} / \mathrm{dL}$ had malignancy, whereas only $16 / 571$ (2.8\%) with hemoglobin levels $>13 \mathrm{~g} / \mathrm{dL}$ had malignancy $(p<0.01)$. Alcohol in 20 (40\%) and smoking in 19 (38\%) patients were found to have an association with malignancy $(p<0.05)$.

\section{Characterization of alarm features}

Alarm features were present in $206(22.9 \%)$, out of whom malignant lesions were seen in $46(22.3 \%)$ patients (Table 2). Weight loss was the most common alarm feature reported in $116(12.9 \%)$ patients. The PPV, NPV, sensitivity, and specificity of finding a
Table 2 Frequency of alarm features in dyspeptic patients with or without malignancy

\begin{tabular}{llc}
\hline Alarm features & $\begin{array}{l}\text { Dyspepsia with malignancy, } \\
n=50, n(\%)^{\#}\end{array}$ & $\begin{array}{l}\text { Dyspepsia without malignancy, } \\
n=850, n(\%)^{\#}\end{array}$ \\
\hline Any alarm feature & $46(92)$ & $160(18.8)$ \\
Weight loss & $38(76)$ & $78(9.1)$ \\
Dysphagia & $19(38)$ & $33(3.8)$ \\
Anemia & $19(38)$ & $60(7)$ \\
Persistent vomiting & $12(24)$ & $28(3.3)$ \\
Gastrointestinal bleed & $7(14)$ & $37(4.3)$ \\
Abdominal mass & $5(10)$ & $1(0.1)$ \\
Family history of malignancy & $5(10)$ & $8(0.9)$ \\
Supraclavicular lymph node & $2(4)$ & $2(0.2)$ \\
\hline
\end{tabular}

${ }^{\text {\# }}$ Some patients may have more than one alarm feature 
Table 3 Positive predictive value, negative predictive value, sensitivity, and specificity of alarm features

\begin{tabular}{lllll}
\hline Alarm features & $\begin{array}{l}\text { Positive } \\
\text { predictive } \\
\text { value }(\%)\end{array}$ & $\begin{array}{l}\text { Negative } \\
\text { predictive value } \\
(\%)\end{array}$ & $\begin{array}{l}\text { Sensitivity } \\
(\%)\end{array}$ & $\begin{array}{l}\text { Specificity } \\
(\%)\end{array}$ \\
\hline Any alarm feature & 22.3 & 99.4 & 92 & 81.2 \\
Weight loss & 32.7 & 98.4 & 76 & 90.8 \\
Dysphagia & 36.5 & 96.3 & 38 & 96.1 \\
Persistent vomiting & 30 & 95.5 & 24 & 96.7 \\
Gastrointestinal bleed & 15.9 & 95 & 14 & 95.6 \\
Abdominal mass & 83.3 & 94.9 & 10 & 99.9 \\
Family history of malignancy & 38.5 & 94.9 & 10 & 99.1 \\
Anemia & 24 & 96.2 & 38 & 92.9 \\
Supraclavicular lymph node & 50 & 94.6 & 4 & 99.8 \\
\hline
\end{tabular}

malignancy in patients with various alarm features are given in Table 3. Altogether, the alarm features had a sensitivity of $92 \%$ and specificity of $81.2 \%$ for predicting malignancy. All the alarm features put together had a high NPV of $99.4 \%$ with each alarm feature having a NPV beyond $95 \%$. The sensitivity and specificity for weight loss were $76 \%$ and $90.8 \%$, while that of palpable abdominal mass was $10 \%$ and $99.9 \%$ respectively. Abdominal mass and palpable supraclavicular lymph nodes showed the highest probability of predicting malignancy $(83.3 \%$ [5/6] and 50\% [2/4] respectively). Also the PPV of the alarm features put together increased with increasing age from $8.2 \%$ in the age group of $18-40$ reaching $50 \%$ in those in the age group of 80-100. Based on the ROC curve (Fig. 1), the optimal age for screening of malignancy was 46.5 years with a ROC of 0.763 (fair), sensitivity of $82 \%$, and specificity of
$60 \%$. When the age was combined with alarm features, the ROC of 0.914 (excellent) was obtained.

\section{Discussion}

By prospectively analyzing patients with dyspepsia over a 14month period, we found that alarm features are effective tools to identify malignant lesions in these patients. Malignancy was seen in $5.5 \%$ of our patients, while the reported frequency of malignancy in patients with dyspepsia from India varies from $3.9 \%$ to $8.3 \%[12,13]$, In our study, gastric malignancies accounted for $56 \%$, which was somewhat similar to studies conducted across the country, wherein malignant pathology was more often a gastric malignancy $[12,13]$.
Fig. 1 Receiver operating characteristic curve for age and malignancy

\section{ROC Curve}

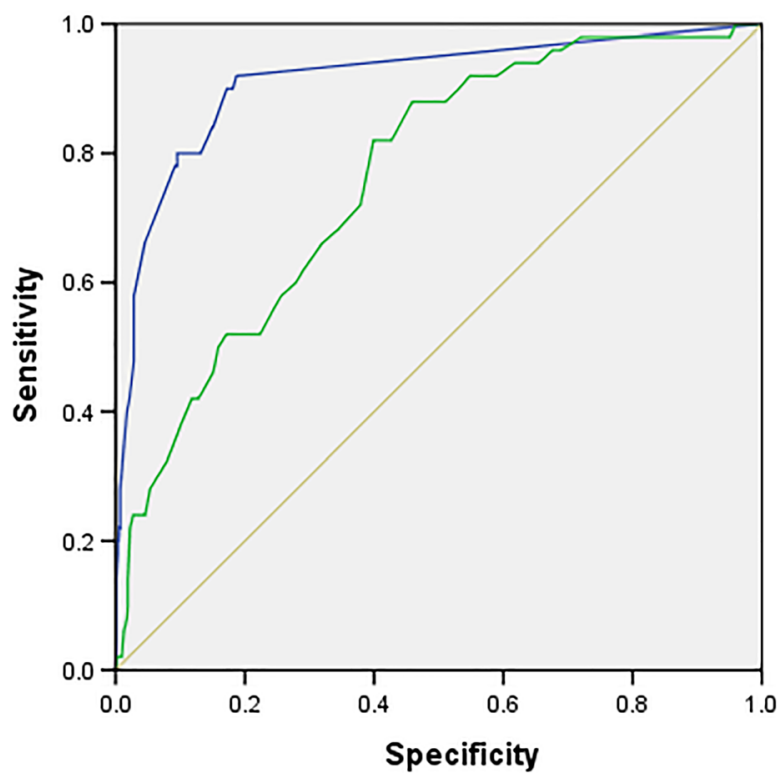

Source of the Curve

- Predicted probability

- age

- Reference Line 
Weight loss was the most common alarm feature seen in $12.9 \%$ of our patients followed by dysphagia (5.8\%), while palpable supraclavicular lymph nodes $(0.4 \%)$ was the least common. However, Indian data have reported dysphagia as the most frequent alarm feature seen in up to $10 \%$ patients $[12,13]$. This variation could be attributed to the fact that patients presenting with predominant dysphagia without associated dyspepsia were not part of our study. In patients with alarm symptoms, malignant lesions were found in $22.3 \%$, which was comparable to other studies with a reported frequency of $25 \%$ [12]. Abdominal mass and palpable supraclavicular lymph nodes showed the highest probability of finding a malignancy of $83.3 \%$ and $50 \%$ respectively. The NPV of alarm features altogether was $99.4 \%$ with each of the individual alarm features having NPV of $95 \%$ or greater similar to that seen in another study [14], which makes it very attractive with little chance of missing an underlying malignancy.

A few meta-analyses described significant heterogeneity between the studies $[15,16]$. The sensitivity and specificity of alarm symptoms varied significantly from $0 \%$ to $83 \%$ and $40 \%$ to $98 \%$ respectively [15]. The presence of any alarm feature in our study showed a sensitivity of $92 \%$ and a specificity of $81.2 \%$. Among individual alarm features, weight loss had the highest sensitivity of $76 \%$ with a specificity of $90.8 \%$. Anemia and dysphagia had a sensitivity of $38 \%$ each. The rest of the alarm features had a very low sensitivity of $<25 \%$ each of which was almost similar to the data in the meta-analysis by Fransen et al. [16]. There is an increase in the risk of gastric and esophageal cancer with advancing age, but a cutoff age is currently unavailable [17]. The age recommendation for endoscopy in patients with dyspepsia should be decided partly by the incidence of malignancy in that country. The American Gastroenterological Association's position statement recommends a cutoff age of 55 years for endoscopy [18]. Whereas, the Asian guidelines recommend endoscopy in new-onset dyspeptic patients over 40 years of age in areas of high prevalence and over 45 and 50 years in areas of intermediate and low prevalence respectively. Bangladesh, India, and Thailand are considered low-risk countries [19]. The optimal age for malignancy screening was estimated to be 46.5 years in our study with a sensitivity of $82 \%$ and specificity of $60 \%$ with an area under curve (AUC) of 0.763 similar to that reported by Khademi et al. [20] which is far below the cutoff age recommended by Asian or western guidelines. The AUC increased to 0.914 in our study when age was combined with the other alarm features.

We found that dyspeptic patients with underlying malignancy had a shorter mean duration of symptoms ( $<6$ months) when compared with those who did not harbor malignancy, This was statistically significant $(p<0.001$; see the results section). Higher proportion of dyspeptic patients with hemoglobin levels $<10 \mathrm{~g} / \mathrm{dL}(21.3 \%)$ showed presence of malignancies compared with those with hemoglobin levels $>10 \mathrm{~g} / \mathrm{dL}(4.4 \%)(p<0.001)$, making it an ideal hemoglobin level to begin screening for malignancies. The alarm features put together had a very high NPV $(99.4 \%)$ which makes the chances of missing of malignancy was less likely, i.e., 1:166 chance of missing malignancy in those without alarm features. The present study had a few limitations. This was a singlecentered study, with noncomparative design done in a tertiary care referral center; the data may not be representative of the community in general. There may be an element of referral bias. In ideal scenarios, each community should derive their own cutoff age from well-conducted local community studies.

To summarize, alarm features are effective and inexpensive tools to identify malignant lesions in patients with dyspepsia. Patients of age group 40 to 49 years with dyspeptic alarm symptoms (predominantly weight loss) need prompt endoscopy to screen for malignancy. Routine endoscopic surveillance should be limited to patients with alarm features. Alarm symptoms are useful in India and these results are applicable to countries with similar epidemiologic, socioeconomic, and healthcare conditions.

Author contributions AS and GB conducted the study. AS, GB, SS, and CGP were involved in the routine management of these patients. AS was involved in data collection and analysis. AS drafted the manuscript. GB, CGP, and SS gave vital inputs. All authors gave final approval to the version submitted.

Funding Open access funding provided by Manipal Academy of Higher Education, Manipal.

Acknowledgments We would like to thank Department of Pathology at Kasturba Medical College, Manipal for their assistance in reporting histopathology specimens.

\section{Compliance with ethical standards}

Conflict of interest AS, GB, SS, and CGP declare that they have no conflict of interest.

Ethics approval The study was performed in a manner to conform to the Helsinki Declaration of 1975, as revised in 2000 and 2008 concerning human and animal rights. The study protocol was approved by the institutional review board and ethics committee of the coordinating center.

Disclaimer The authors are solely responsible for the data and the contents of the paper. In no way, the Honorary Editor-in-Chief, Editorial Board Members, or the printer/publishers are responsible for the results/ findings and content of this article.

Open Access This article is licensed under a Creative Commons Attribution 4.0 International License, which permits use, sharing, adaptation, distribution and reproduction in any medium or format, as long as you give appropriate credit to the original author(s) and the source, provide a link to the Creative Commons licence, and indicate if changes were made. The images or other third party material in this article are included in the article's Creative Commons licence, unless indicated otherwise in a credit line to the material. If material is not included in the article's 
Creative Commons licence and your intended use is not permitted by statutory regulation or exceeds the permitted use, you will need to obtain permission directly from the copyright holder. To view a copy of this licence, visit http://creativecommons.org/licenses/by/4.0/.

\section{References}

1. Graham DY, Rugge M. Clinical practice: diagnosis and evaluation of dyspepsia. J Clin Gastroenterol. 2010;44:167-72.

2. Oustamanolakis P, Tack J. Dyspepsia: organic versus functional. J Clin Gastroenterol. 2012;46:175-90.

3. Talley NY, Vakil NB, Moayyedi P. American Gastroenterological Association technical review on the evaluation of dyspepsia. Gastroenterology. 2005;129:1756-80.

4. Ghoshal UC, Singh R, Chang FY, et al. Epidemiology of uninvestigated and functional dyspepsia in Asia: facts and fiction. J Neurogastroenterol Motil. 2011;17:235-44.

5. Lacy BE, Weiser KT, Kennedy AT, et al. Functional dyspepsia: the economic impact to patients. Aliment Pharmacol Ther. 2013;38: $170-7$.

6. Schwartz MD. Dyspepsia, peptic ulcer disease, and esophageal reflux disease. West J Med. 2002;176:98-103.

7. Kitapçioğlu G, Mandiracioğlu A, Caymaz Bor C, Bor S. Overlap of symptoms of dyspepsia and gastroesophageal reflux in the community. Turk J Gastroenterol. 2007;18:14-9.

8. Brun R, Kuo B. Functional dyspepsia. Ther Adv Gastroenterol. 2010;3:145-64.

9. Bai Y, Li ZS, Zou DW, et al. Alarm features and age for predicting upper gastrointestinal malignancy in Chinese patients with dyspepsia with high background prevalence of Helicobacter pylori infection and upper gastrointestinal malignancy: an endoscopic database review of 102,665 patients from 1996 to 2006 . Gut. 2010;59:7228 .

10. Drossman DA. The functional gastrointestinal disorders and the Rome III process. Gastroenterology. 2006;130:1377-90.
11. Karamanolis GP, Tack J. Current management of functional dyspepsia: impact of Rome III subdivision. Ann Gastroenterol. 2012;25:96-9.

12. Adlekha S, Chadha T, Krishnan P, Sumangala B. Prevalence of helicobacter pylori infection among patients undergoing upper gastrointestinal endoscopy in a medical college hospital in Kerala, India. Ann Med Health Sci Res. 2013;3:559-63.

13. Sumathi B, Navaneethan U, Jayanthi V. Appropriateness of indications for diagnostic upper gastrointestinal endoscopy in India. Singap Med J. 2008;49:970-6.

14. Saha AK, Maitra S, Hazra SC. Epidemiology of gastric cancer in the Gangetic areas of West Bengal. ISRN Gastroenterol. 2013;2013:823483.

15. Vakil N, Moayyedi P, Fennerty MB, Talley NJ. Limited value of alarm features in the diagnosis of upper gastrointestinal malignancy: systematic review and meta-analysis. Gastroenterology. 2006;131:390-401.

16. Fransen GA, Janssen MJ, Muris JW, et al. Meta-analysis: the diagnostic value of alarm symptoms for upper gastrointestinal malignancy. Aliment Pharmacol Ther. 2004;20:1045-52.

17. Maconi G, Manes G, Porro GB. Role of symptoms in diagnosis and outcome of gastric cancer. World J Gastroenterol. 2008;14:1149 55 .

18. Talley NJ, Vakil N. Practice parameters committee of the American College of Gastroenterology. Guidelines for the management of dyspepsia. Am J Gastroenterol. 2005;100:2324-37.

19. Miwa H, Ghoshal UC, Gonlachanvit S, et al. Asian consensus report on functional dyspepsia. J Neurogastroenterol Motil. 2012;18: $150-68$.

20. Khademi H, Radmard AR, Malekzadeh F, et al. Diagnostic accuracy of age and alarm symptoms for upper GI malignancy in patients with dyspepsia in a GI clinic: a 7-year cross-sectional study. PLoS One. 2012;7:e39173.

Publisher's note Springer Nature remains neutral with regard to jurisdictional claims in published maps and institutional affiliations. 